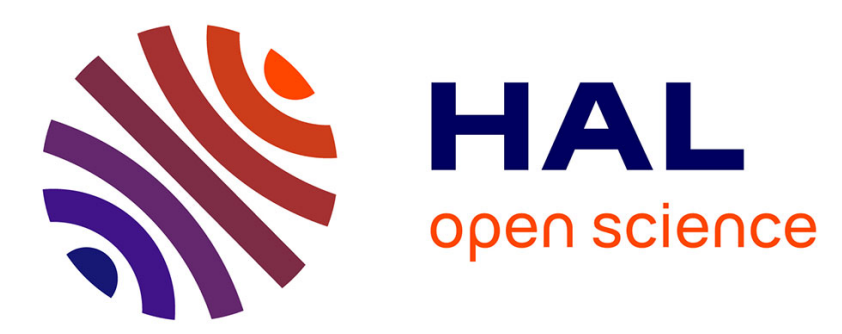

\title{
Détermination temporelle et spatiale de la fonction de distribution en énergie des électrons dans une post-décharge
}

\author{
P. Monchicourt, M. Ahrweiller, R. Deloche
}

\section{- To cite this version:}

P. Monchicourt, M. Ahrweiller, R. Deloche. Détermination temporelle et spatiale de la fonction de distribution en énergie des électrons dans une post-décharge. Revue de Physique Appliquée, 1979, 14 (2), pp.451-460. 10.1051/rphysap:01979001402045100 . jpa-00244614

\section{HAL Id: jpa-00244614 https://hal.science/jpa-00244614}

Submitted on 1 Jan 1979

HAL is a multi-disciplinary open access archive for the deposit and dissemination of scientific research documents, whether they are published or not. The documents may come from teaching and research institutions in France or abroad, or from public or private research centers.
L'archive ouverte pluridisciplinaire HAL, est destinée au dépôt et à la diffusion de documents scientifiques de niveau recherche, publiés ou non, émanant des établissements d'enseignement et de recherche français ou étrangers, des laboratoires publics ou privés. 


\title{
Détermination temporelle et spatiale de la fonction de distribution en énergie des électrons dans une post-décharge
}

\author{
P. Monchicourt, M. Ahrweiller et R. Deloche \\ Service de Physique Atomique, Centre d'Etudes Nucléaires de Saclay, \\ B.P. ${ }^{\circ} 2,91190$ Gif-sur-Yvette, France \\ (Reçu le 12 juillet 1978, révisé le 25 octobre 1978, accepté le 27 octobre 1978)
}

\begin{abstract}
Résumé. - Afin d'obtenir directement, de façon précise, dans une post-décharge, la fonction de distribution en énergie des électrons avec une bonne résolution spatiale, temporelle et énergétique, un dispositif de mesure de haute sensibilité, par sonde de Langmuir, a été construit et est ici présenté.

Il s'applique à n'importe quel type de décharge répétitive et permet de mesurer, à des pressions relativement élevées, la partie haute énergie des fonctions de distribution.
\end{abstract}

\begin{abstract}
In order to determine experimentally, in an afterglow, the electron energy distribution functions with a very high spatial, temporal and energetic resolution, a very sensitive electronic device has been built and is presented.

It can be used with any type of repetitive discharge and allows measurements of the high energy tail of the distribution functions, even at intermediate pressures.
\end{abstract}

1. Introduction. - La fonction de distribution en énergie des électrons varie en chaque point de l'espace et à chaque instant au cours de la post-luminescence d'un gaz ionisé. Elle ne suit généralement pas la loi de Maxwell-Boltzmann; les électrons ne sont pas à l'équilibre thermodynamique à une température électronique donnée $T_{\mathrm{e}}$ si la désexcitation collisionnelle des états métastables atomiques ou moléculaires crée des électrons de grande énergie en nombre important ou s'il existe toute autre source d'électrons non thermiques. Ces électrons de grande énergie modifient constamment la distribution des vitesses de façon très variable en fonction du temps et de l'espace. C'est ce que l'on observe au cours des post-décharges des gaz rares et plus particulièrement de l'hélium ionisé pour lequel ces distributions ont récemment été calculées de façon précise $[1,2,3]$ à l'aide de modèles numériques, applicables dans d'autres cas et pour d'autres gaz. La détermination expérimentale de l'énergie des électrons dans une post-décharge est donc généralement difficile pour les raisons que nous venons d'évoquer :

- écart à la fonction de Maxwell;

- variation temporelle et spatiale de la distribution.

C'est ainsi que de nombreux auteurs préfèrent supposer la fonction de distribution maxwellienne et faire une évaluation théorique de la température électronique équivalente. D'autres au contraire mettent en ouvre des diagnostics très élaborés qui permettent de mesurer l'énergie moyenne du gaz d'électrons dans tout le volume du gaz ionisé [3,4]. On peut citer, par exemple, la détermination de la fréquence totale de collision électron-atome et électron-ion par interférométrie U.H.F. ou la mesure de la température de rayonnement moyenne des électrons par radiométrie U.H.F. De ces grandeurs macroscopiques moyennes on peut déduire un certain nombre d'informations intéressantes mais on ne peut en tirer les fonctions de distribution en énergie proprement dites.

Afin d'obtenir directement de façon précise la fonction de distribution en énergie des électrons avec une bonne résolution spatiale, temporelle et énergétique nous avons construit un dispositif de mesure par sondes de Langmuir.

Ce diagnostic, assez souvent utilisé dans le passé pour la détermination des fonctions de distribution en énergie des électrons, en décharge continue, n'a précédemment été mis en œuvre avec succès, au cours de la post-lumínescence d'une décharge pulsée, qu'une seule fois à notre connaissance par Blagoev et al. $[5,6]$ dans l'hélium ionisé à basse pression (1 torr).

Nous présentons ici un ensemble expérimental, fondé sur le même principe mais pouvant être utilisé à plus haute pression (5 à 100 torr), avec une sensibilité 
beaucoup plus grande, une excellente résolution temporelle et une résolution spatiale permettant de définir les profils spatiaux des électrons à une énergie donnée. Ce diagnostic s'applique à n'importe quel type de décharge répétitive, même si des impulsions de tension, de plusieurs kilovolts, sont nécessaires pour créer le gaz ionisé.

Nous montrons qu'un tel diagnostic permet de mesurer, en valeur absolue avec précision, la partie haute énergie de la fonction de distribution même à pression relativement élevée et nous donnons ses limites de validité. On ne peut en général atteindre la température équivalente de la partie maxwellienne des distributions dans ces conditions de pression. Quelques résultats expérimentaux, obtenus dans l'hélium ionisé en régime de relaxation à 5 et 10 torr sont également présentés pour illustrer les performances de notre dispositif expérimental.

Nous montrerons dans un prochain article [7] comment ces courbes ont été interprétées à l'aide de modèles mathématiques numériques, afin de déterminer entre autres quels sont les mécanismes de création des électrons non maxwelliens et de mesurer directement leur section efficace.

La finalité de ce travail était en effet de déterminer l'ensemble des mécanismes élémentaires qui gouvernent l'évolution de la post-décharge de l'hélium ionisé, afin d'en déduire plus précisément quels sont les mécanismes de recombinaison de l'ion $\mathrm{He}_{2}^{+}$.

2. Principe de la mesure. - La fonction de distribution en énergie des électrons $f(\varepsilon)$ est déduite de la mesure de la dérivée seconde du courant électronique par rapport à la tension, suivant la formule de Druyvesteyn [8]

$i^{\prime \prime}(v)=\frac{1}{4} e S \sqrt{\frac{2 e}{m}} \frac{f(\varepsilon)}{\varepsilon^{1 / 2}}$

$i^{\prime \prime}(v)=2,373 \times 10^{-12} S \frac{f(\varepsilon)}{\varepsilon^{1 / 2}}$ en $\mathrm{A} \cdot \mathrm{V}^{-2}$

où $f(\varepsilon)$ est exprimée en $\mathrm{cm}^{-3}(\mathrm{eV})^{-1}, e$ et $m$ représentent la charge et la masse de l'électron, $S$ la surface de la sonde en $\mathrm{cm}^{2}$ et où $\varepsilon$ est l'énergie, en $\mathrm{eV}$, correspondant à la différence de potentiel imposée entre la sonde et le gaz ionisé.

La formule (1) s'applique à toute sonde convexe et à toute fonction de distribution isotrope, pourvu que le libre-parcours moyen des électrons $\lambda_{\mathrm{e}}$ soit grand devant le rayon « $a$ » de la sonde et devant l'épaisseur de la gaine ionique qui l'entoure.

A basse pression, dans un gaz faiblement ionisé la formule (1) est parfaitement valable. Si l'on travaille à pression plus élevée, $\lambda_{\mathrm{e}}$ devient de l'ordre de « $a$ », alors la formule de Druyvesteyn (1) doit être modifiée en appliquant l'une des nombreuses théories de sonde [9] élaborées pour ces pressions intermédiaires où $\lambda_{\mathrm{e}} \sim a$. Il est démontré que la formule (1) est applicable tant que le nombre d'électrons collectés par la sonde reste faible devant le nombre d'électrons repoussés.

Dans le cas d'une distribution de Maxwell, caractérisée par une température $T_{\mathrm{e}}$, Case [10] a donné une expression de la dérivée seconde du courant électronique en fonction du rapport $a / \lambda_{\mathrm{e}}$, sous une forme analytique simple :

$$
i^{\prime \prime}(v)=\frac{1}{4} e S \sqrt{\frac{2 e}{m}} \frac{f(\varepsilon)}{\varepsilon^{1 / 2}} \frac{1}{1+\frac{a}{\lambda_{\mathrm{e}}} I_{\mathrm{e} 0} \exp \left(-e V / k T_{\mathrm{e}}\right)}
$$

où $I_{\mathrm{e} 0}$ est une constante comprise entre 1 et 3 .

Si $\rho$ désigne le rapport du nombre d'électrons collectés au nombre d'électrons repoussés, les formules (1) et (2) coïncident à mieux de $1 \%$ près, tant que le produit $\rho a / \lambda_{\mathrm{e}}$ reste inférieur à $10^{-2}$. Cette condition est toujours remplie entre 5 et 20 torr dans l'hélium ionisé, pour des électrons d'énergie supérieure à $1 \mathrm{eV}$. Si l'écart à la distribution de Maxwell est limité, l'utilisation de la formule (1) de Druyvesteyn conduit à une très faible erreur systématique pour la détermination de la partie haute énergie de la fonction de distribution. Pour la partie basse énergie les formules (1) .et (2) ne sont équivalentes que si $a / \lambda_{\mathrm{e}} \ll 1$.

La mesure de la dérivée seconde est obtenue en adaptant au régime impulsionnel une technique bien connue en régime continu [11].

Ce principe de mesure a déjà été appliqué à basse pression dans une post-décharge d'hélium par Blagoev et al. $[5,6]$.

L'analyse temporelle du signal répétitif cherché se fait en appliquant sur la sonde, à un instant $t_{0}$ choisi au cours de la post-luminescence, une brève impulsion de tension $V+\Delta V$, de durée $\Delta t$ petite devant la période de répétition $T=2 \Pi / \Omega$ de création du gaz ionisé. Cette méthode consiste à superposer à la tension $V$ de polarisation de la sonde un signal sinusoïdal $\Delta V$ de pulsation $\omega_{2}$, modulé en amplitude à la pulsation $\omega_{1}$ :

$\Delta V=V_{0}\left(1+\cos \omega_{1} t\right) \cos \omega_{2} t f(t)$

avec $\omega_{1} \ll \omega_{2}$ et

$f(t)=\left\{\begin{array}{l}1 \text { pour } n T-\frac{\Delta t}{2}<t<n T+\frac{\Delta t}{2} \\ 0 \text { par ailleurs }\end{array}\right.$

La non-linéarité du plasma fait apparaître dans le courant de sonde $i(V+\Delta V)$, une composante $i_{\omega_{1}}(V)$ à la fréquence $\omega_{1} / 2 \Pi$.

$$
\begin{aligned}
& i(V+\Delta V)=i(V)+\frac{\Delta V}{1 !} i^{\prime}(V)+\frac{\Delta V^{2}}{2 !} i^{\prime \prime}(V)+\cdots \\
& i_{\omega_{1}}(V)=\frac{\Delta t}{T}\left[\frac{1}{2} V_{0}^{2} i^{\prime \prime}(V)+\frac{7}{64} V_{0}^{4} i^{I V}(V)+\cdots\right] \cos \omega_{1} t
\end{aligned}
$$


Si l'amplitude de modulation $V_{0}$ est petite :

$i_{\omega_{1}}(V)=\frac{\Delta t}{T} \frac{V_{0}^{2}}{2} i^{\prime \prime}(V) \cos \omega_{1} t$.

A l'aide d'une détection sélective à la fréquence $\omega_{1} / 2 \Pi$ on mesure le courant $i_{\omega_{1}}(V)$, d'où l'on tire la dérivée seconde du courant électronique de sonde $i^{\prime \prime}(V)$ (formule (6)), on en déduit enfin la fonction de distribution $f(\varepsilon)$ (formule (1)) de façon beaucoup plus précise que par double différenciation graphique ou électronique de $i(V)$.

En pratique on relève, à chaque instant choisi au cours de la post-luminescence, les valeurs de $i_{\omega 1}$ en fonction de la tension de polarisation $V$ que l'on fait lentement varier, entre 0 et $-20 \mathrm{~V}$ par exemple, par incrémentation de faible amplitude.

Le taux d'échantillonnage $\Delta t / T$ et l'amplitude de modulation $V_{0}$ sont choisis afin d'optimiser

- le rapport signal sur bruit,

- la résolution temporelle,

- la résolution en énergie.

L'utilisation consécutive de plusieurs sondes, disposées en différents points du gaz ionisé, permet la détermination des distributions spatiales des électrons de même énergie.

3. Dispositif expérimental. - 3.1 LE DISPOSITIF ÉLECTRONIQUE. - Le dispositif électronique doit permettre de mesurer un courant $i_{\omega_{1}}(V)$ extrêmement petit, à une pulsation $\omega_{1}$ bien déterminée.

La valeur de ce courant dépend du nombre d'électrons collectés par la sonde, à un instant donné de la post-luminescence, pendant une durée $\Delta t$, pour une tension de polarisation négative $V$ et pour une modulation d'amplitude $V_{0}$, qui détermine la résolution de la mesure en énergie.

La composante $i_{\omega_{1}}(V)$ du courant doit être extraite du courant ionique intense, du bruit très important et des autres composantes du courant électronique.

Le dispositif électronique doit donc être très sélectif en fréquence et d'une très grande sensibilité.

Il doit d'autre part n'être composé que d'éléments parfaitement linéaires pour ne pas introduire d'erreur systématique importante ou simplement interdire toute mesure. Autrement dit en l'absence de plasma aucun signal de pulsation $\omega_{1}$ ne doit être détectable ce qui impose également un signal de modulation $\Delta V$ ne comprenant que les pulsations $\omega_{2}, \omega_{2}-\omega_{1}$ et $\omega_{2}+\omega_{1}$.

La conception du dispositif électronique doit être adaptée aux conditions particulières de chaque expérience, mais de façon générale, au cours d'une postdécharge, le circuit électronique de mesure doit répondre à certaines caractéristiques et satisfaire à un certain nombre de contraintes :

1) Une bonne résolution en énergie demande une très grande sensibilité.
2) La création de décharges de courte durée à intervalles de temps réguliers, dans un gaz à pression relativement élevée, exige souvent des impulsions de haute tension, dont la valeur dépend également de la géométrie de la cellule. Il est donc indispensable de protéger le circuit de sonde pendant la durée de ces impulsions.

3) La période de répétition $T$ du plasma est imposée le plus souvent par les conditions expérimentales. Elle dépend du gaz étudié, et doit être d'autant plus grande que la pression est élevée, les constantes de temps de décroissance des paramètres principaux augmentant avec la densité des neutres. La période $T$ est également souvent imposée par des considérations pratiques de stabilité, de symétrie, ou de centrage de la décharge. Il faut alors choisir les pulsations $\omega_{1}$ et $\omega_{2}$ en fonction de $T$.

4) Une bonne résolution temporelle exige d'autre part que la durée $\Delta t$ de polarisation et de modulation en tension de la sonde soit, non seulement courte devant la période $T$, mais également par rapport à la constante de temps $\mathfrak{C}$ de décroissance de l'énergie des électrons.

5) Enfin la réponse du plasma à l'impulsion de tension modulée $V+\Delta V$, pendant le temps $\Delta t$ de l'ouverture de la porte de mesure, n'est pas instantanée. Elle n'apparaît qu'après un temps $\theta$ correspondant à la durée de formation de la gaine ionique autour de la sonde [13]. La pulsation $\omega_{2}$ de la porteuse doit être très inférieure à $2 \Pi / \theta$.

Ces différentes considérations conduisent à la suite des inégalités suivantes :

$0<\omega_{1}<\frac{2 \Pi}{T}<\frac{2 \Pi}{6}<\frac{2 \Pi}{\Delta t}<\omega_{2}<\frac{2 \Pi}{\theta}$

en effet, la détection sélective à la pulsation $\omega_{1}$ doit se faire avec un taux de réjection très élevé pour toutes les autres fréquences, en particulier pour $\omega_{2}$ et les harmoniques : $n \frac{2 \Pi}{T}$, très proches les uns des autres.

Si l'on choisit $\omega_{1}$ supérieur à $2 \Pi / T$ et compris entre deux harmoniques consécutifs, il est pratiquement très difficile d'obtenir une réjection suffisante.

Le dispositif que nous avons construit est représenté figure 1, il comprend les éléments suivants, montés en série, entre la sonde et la masse :

1) Le commutateur haute tension, dont le seul rôle est de protéger le circuit de sonde pendant la durée de la décharge haute tension. Une ampoule Reed rapide, Hamelin $5 \mathrm{kV}$ ouvre le circuit pendant la décharge. Ce contacteur à lame souple, commandé par une bobine, permet l'analyse de l'énergie des électrons au cours de la post-luminescence, environ $500 \mu$ s après la fin de la décharge en raison des rebondissements de la lame à la fermeture. Nous avons dû renoncer à utiliser des transistors haute tension, montés en push-pull, qui ne peuvent commuter 


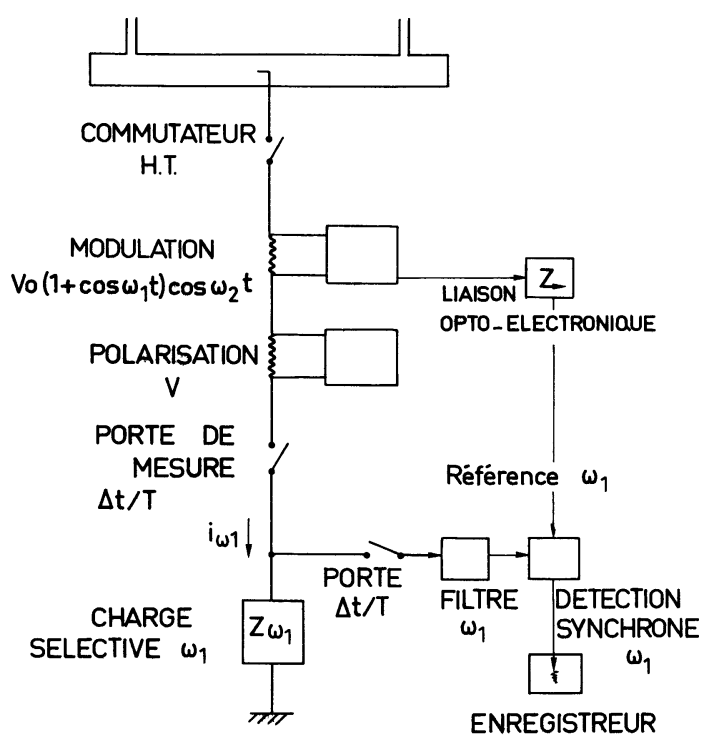

Fig. 1. - Schéma du circuit électronique de sonde.

[Scheme of the probe electronic circuit.]

les très faibles courants collectés par la sonde et introduisent une légère non-linéarité.

2) La polarisation $V$ (Fig. 2), réalisée à l'aide de piles, elle porte la sonde à un potentiel réglable de $-30 \mathrm{~V}$ à $+30 \mathrm{~V}$.

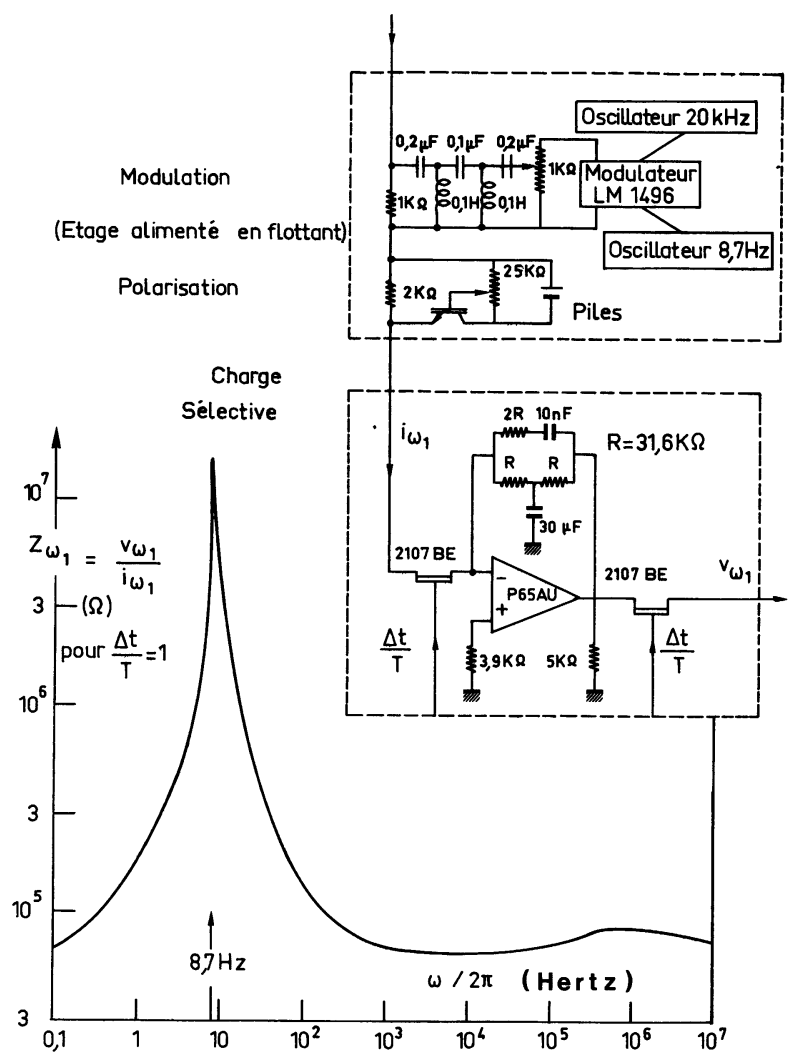

Fig. 2. - Circuit électronique de génération de la tension modulée appliquée sur la sonde, présenté avec le circuit de la charge sélective. La variation de l'impédance sélective du circuit de mesure en fonction de la fréquence montre un pic de résonance important à $8,7 \mathrm{~Hz}$.

[Tension modulator and selective charge. The variation of the selective impedance of the circuit as a function of the frequency is shown. It presents an important resonance peak at $8.7 \mathrm{~Hz}$.]
3) La modulation $\Delta V$ (Fig. 2), est appliquée aux bornes d'une résistance de $1 \mathrm{k} \Omega$, par un circuit, à potentiel flottant, alimenté à l'aide d'une batterie d'accumulateurs. Cette solution est préférable à l'utilisation d'un transformateur de liaison, dont la non-linéarité créerait un signal de démodulation à la pulsation $\omega_{1}$, dans des conditions d'autant plus difficiles que l'on travaillerait à basse fréquence.

Le modulateur a été réalisé à partir de deux oscillateurs sinusoïdaux Frequency Device, modèles 442 et 444 , de grande stabilité, dont les fréquences réglables ont été fixées à $20 \mathrm{kHz}$ pour $\omega_{2}$ et $8,7 \mathrm{~Hz}$ pour $\omega_{1}$, à mieux de $10^{-4}$ près. Le modulateur proprement dit (circuit intégré National LM 1496) comprend le réglage de la profondeur de modulation, fixée expérimentalement à $98,5 \%$ dans toutes nos mesures, pour obtenir la sensibilité maximale tout en évitant toute détection d'un signal parasite à la fréquence $\omega_{1}$ en l'absence de plasma. Un étage Darlington attaque le circuit de sonde à basse impédance $(1 \mathrm{k} \Omega)$. Afin de n'introduire aucun signal à $8,7 \mathrm{~Hz}$ cette attaque se fait par l'intermédiaire d'un filtre LC passe-haut, de $1 \mathrm{kHz}$ de fréquence de coupure.

Enfin un système opto-électronique (H.P. 50824360) transmet à la détection synchrone (PAR H.R. 8) un signal de référence de pulsation $\omega_{1}$ parfaitement découplé du modulateur.

L'amplitude de modulation $V_{0}$ est réglable entre 0 et $1 \mathrm{~V}$ à $10^{-2} \mathrm{~V}$ près.

4) La porte de mesure (porte à effet de champ Amelco 2107BE) détermine, à la microseconde près, l'instant $t$ de la fermeture du circuit et la durée $\Delta t$ de la mesure (Fig. 3), c'est-à-dire de l'application de la tension modulée sur la sonde.

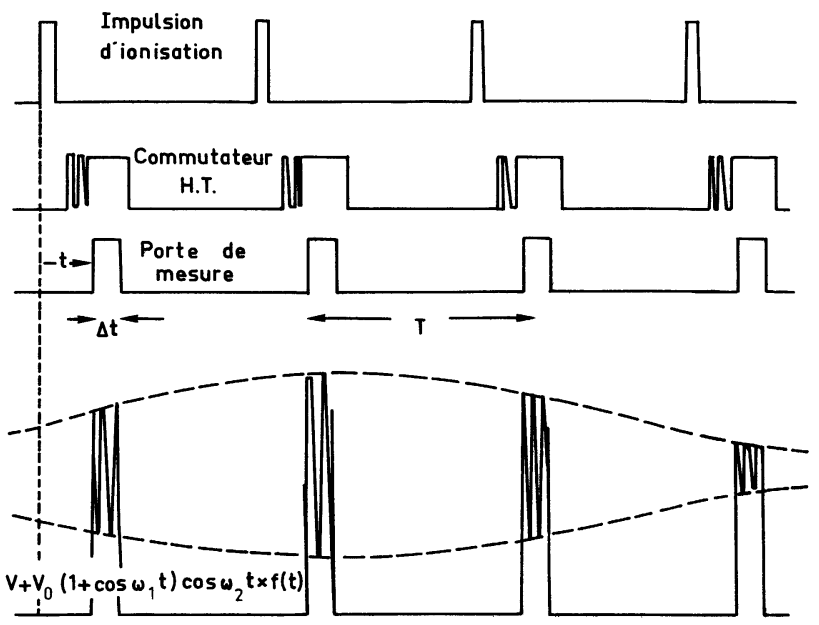

Fig. 3. - Séquence temporelle des impulsions et représentation de la tension modulée de la sonde.

[Pulse time sequence and representation of the modulated tension of the probe.]

5) La charge sélective (Figs. 1 et 2), permet la mesure du courant $i_{\omega_{1}}$ extrêmement faible, de l'ordre du picoampère à la pulsation $\omega_{1}$. Elle doit être de très 
forte impédance à la fréquence $\omega_{1} / 2 \Pi$ et tendre vers zéro partout ailleurs. Elle est constituée d'un amplificateur à contre réaction sélective (double $T$ ponté), centrée sur $8,7 \mathrm{~Hz}$. Cette charge ferme le circuit de sonde sur la masse. Son impédance de transfert à la résonance (Fig. 2) est $Z_{\omega_{1}}=15 \mathrm{M} \Omega$ et sa bande passante à mi-hauteur de $1 \mathrm{~Hz}$.

6) La détection (Fig. 1). Afin de réduire le taux de parasites observés sans nuire au rapport signal/bruit de l'ensemble de la chaîne, une seconde porte, identique à la première, et commandée par le même signal, est placée en aval de la charge sélective. Cette porte échantillonne à la pulsation $\Omega \gg \omega_{1}$ le signal sinusoïdal de pulsation $\omega_{1}$ et d'amplitude proportionnelle à $\Delta t / T$ (formule (6) et Fig. 3). Elle transmet un signal qui est donc proportionnel à $(\Delta t / T)^{2}$ (Fig. 4).
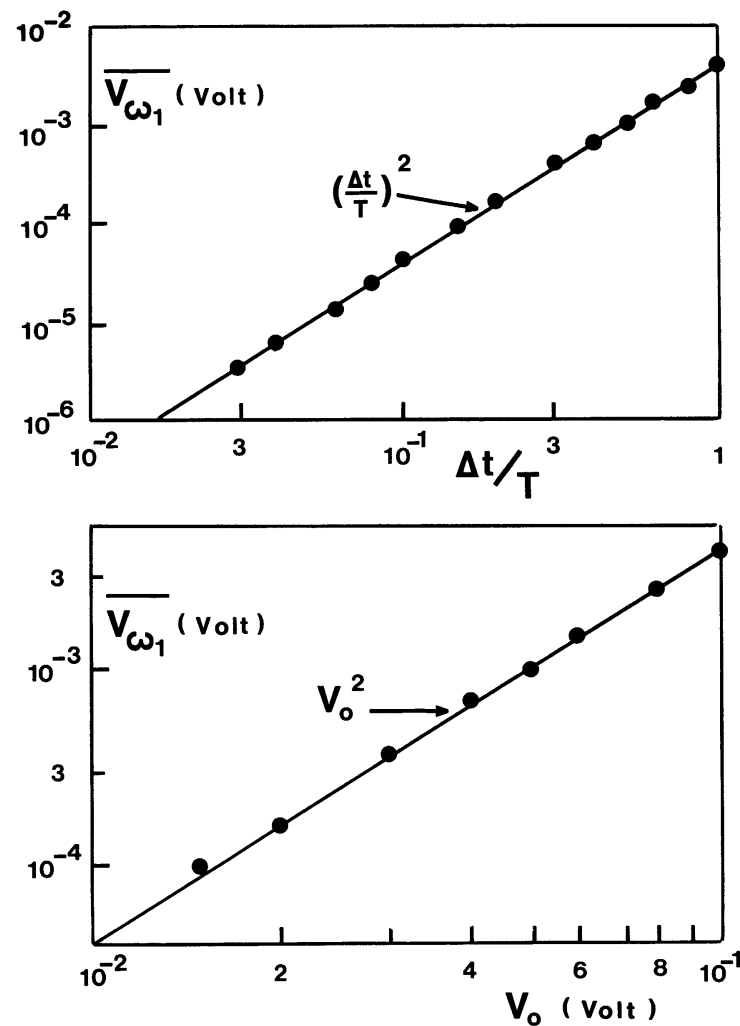

Fig. 4. - Réponse impulsionnelle du circuit de mesure en fonction du rapport $\Delta t / T$ et de l'amplitude de polarisation $V_{0}$. Le signal mesuré est proportionnel à $(\Delta t / T)^{2}$ et à $V_{0}^{2}$.

[Impulse response of the electronic circuit as a function of $\Delta t / T$ and polarisation amplitude $V_{0}$. The measured signal is proportional to $(\Delta t / T)^{2}$ and $\left.V_{0}^{2} \cdot\right]$

Un filtre actif passe-bande (Teltron TAF 5002), ayant un taux de réjection à très forte pente $(24 \mathrm{~dB}$ par octave) permet d'isoler la composante du courant à $8,7 \mathrm{~Hz}$.

Enfin une détection synchrone (PAR HR 8) réalise une nouvelle amplification sélective du signal, avec une bande passante de l'ordre du centième de hertz et donne un signal continu (avec des constantes de temps d'intégration de l'ordre de $30 \mathrm{~s}$ ) proportionnel à la dérivée seconde cherchée :

$v_{\omega_{1}}=Z_{\omega_{1}} \frac{V_{0}^{2}}{2}\left(\frac{\Delta t}{T}\right)^{2} i^{\prime \prime}(V) \mathcal{C}_{\mathrm{F}} \mathcal{C}_{\mathrm{DS}}$

où $Z_{\omega_{1}}$ est l'impédance de la charge sélective à la pulsation $\omega_{1}$.

$\mathfrak{C}_{\mathrm{F}}$ et $\mathfrak{C}_{\mathrm{DS}}$ sont les coefficients de transmission du filtre et de la détection synchrone

$Z_{\omega_{1}} \mathfrak{G}_{\mathrm{F}} \mathfrak{C}_{\mathrm{DS}}=15 \times 0,52 \times 0,44=3,4 \mathrm{M} \Omega$.

La non-linéarité résiduelle du circuit est très faible. Elle a été mesurée en remplaçant le plasma par une résistance pure. Elle équivaut pour $\Delta t / T=1$ et $V_{0}=1 \mathrm{~V}$ à un courant $i_{\omega_{1}}=3 \times 10^{-12} \mathrm{~A}$.

La réponse impulsionnelle du circuit à une nonlinéarité stationnaire, simulant le plasma, vérifie parfaitement la formule (6) en amont de la seconde porte et la formule (8) en aval (Fig. 4).

Grâce à ces caractéristiques la limite de sensibilité est de l'ordre de $10^{5}$ électrons par $\mathrm{cm}^{3}$ et par $\mathrm{eV}$, pour $\Delta t / T=10^{-2}$ et $V_{0}=1 \mathrm{~V}$.

3.2 LA CEllule eXPÉrimentale. - Les sondes doivent être de petites dimensions par rapport au volume du gaz ionisé afin de n'apporter qu'une très faible perturbation.

Chaque passage de sonde doit être parfaitement isolé et la surface collectrice $S$ doit être bien définie et aussi grande que possible. Il est par contre recommandé que le diamètre des sondes soit très fin si l'on veut travailler à haute pression.

Les sondes doivent être adaptées à toutes les formes de dispositif expérimental et à toutes les conditions de mesure.

Nous avons choisi dans notre cas une cellule cylindrique en pyrex de $40 \mathrm{~cm}$ de longueur et de $1,832 \mathrm{~cm} \pm 0,002 \mathrm{~cm}$ de diamètre intérieur (Fig. 5).

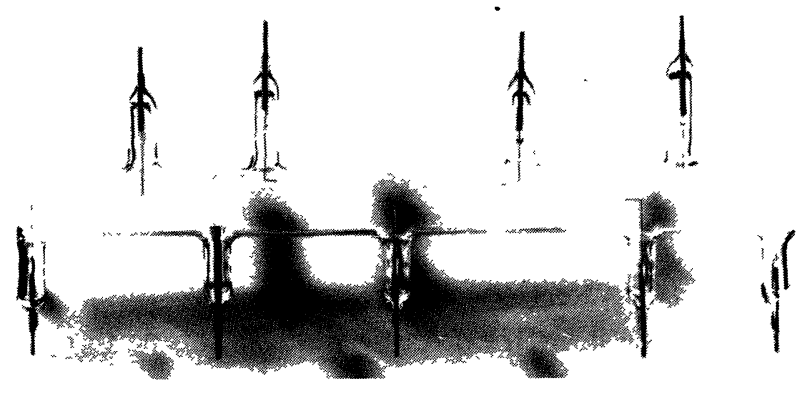

Fig. 5. - Photographie des sondes.

[Photograph of the probes.]

L'isolement électrique est résolu par l'utilisation du pyrex qui permet l'observation optique de la décharge, de son centrage et le contrôle de la symétrie de révolution du gaz ionisé au cours de la post-luminescence. 
Huit sondes cylindriques en tungstène de $0,1 \mathrm{~mm}$ de diamètre et de $5,0 \mathrm{~mm}$ de longueur ont été utilisées. Elles sont toutes parallèles à une directrice du cylindre et situées dans un même plan médian :

- quatre sont suivant l'axe de la cellule pour vérifier l'homogénéité longitudinale du gaz ionisé;

— deux sont placées au milieu du rayon : $R / 2$;

- une au quart du rayon $R / 4$, à partir de l'axe;

- une aux trois quarts du rayon $3 R / 4$, à partir de l'axe.

La surface collectrice de chacune de ces huit sondes est égale à $S=1,5 \pm 0,3 \mathrm{~mm}^{2}$.

Une sonde plane, circulaire, de surface $S=1,77 \pm 0,06 \mathrm{~mm}^{2}$ est enfin positionnée au niveau de la paroi interne du tube pour déterminer la distribution des vitesses du faible flux d'électrons atteignant les parois de l'enceinte.

Le diamètre de l'enrobage en pyrex de la partie radiale, non collectrice, des sondes est inférieur à $0,5 \mathrm{~mm}$.

Un générateur d'impulsions de haute tension, de durée, de taux de répétition et d'amplitude réglables [12], permet de créer la décharge entre deux électrodes en tantale. Ces électrodes sont très rapidement remises à un potentiel nul à la fin de chaque impulsion de tension. Elles ne doivent pas être trop petites devant le volume du gaz ionisé, afin que le potentiel plasma soit toujours bien déterminé au cours de la post-décharge. Il est également important que ces électrodes ne soient pas séparées du volume du gaz ionisé par des passages de trop petit diamètre qui empêchent la fixation du potentiel plasma au cours de la post-décharge.

4. Résultats. - Il est important de connaître la forme de la fonction de distribution en énergie, mais c'est en valeur absolue qu'il faut la mesurer pour en déduire, par exemple, certaines sections efficaces de production d'électrons non thermiques [14].

Afin de calibrer le signal mesuré par la méthode de Druyvesteyn, nous l'avons comparé aux valeurs absolues obtenues par double différenciation graphique de la branche électronique de la caractéristique de sonde $i(V)$, relevée à 5 torr et à $2 \mathrm{~ms}$ dans la postdécharge, au voisinage de la plus forte courbure de la caractéristique de la branche électronique. La coïncidence des deux courbes obtenues (Fig. 6), en utilisant la formule (8) avec $Z_{\omega 1} \mathfrak{C}_{\mathrm{F}} \mathfrak{C}_{\mathrm{DS}}=3,4 \mathrm{M} \Omega$, confirme la validité de la méthode et de l'évaluation de l'impédance caractéristique. Les barres d'erreur d'environ $20 \%$ montrent l'imprécision de la différenciation graphique.

Pour illustrer les performances du dispositif électronique que nous avons réalisé, nous présentons deux séries de courbes obtenues à 20 torr (Fig. 7 et 8). Nous n'avons pas pu faire des mesures par sonde à pression plus élevée dans la géométrie que nous nous étions imposée, afin d'utiliser l'ensemble des résultats expérimentaux précédemment obtenus :
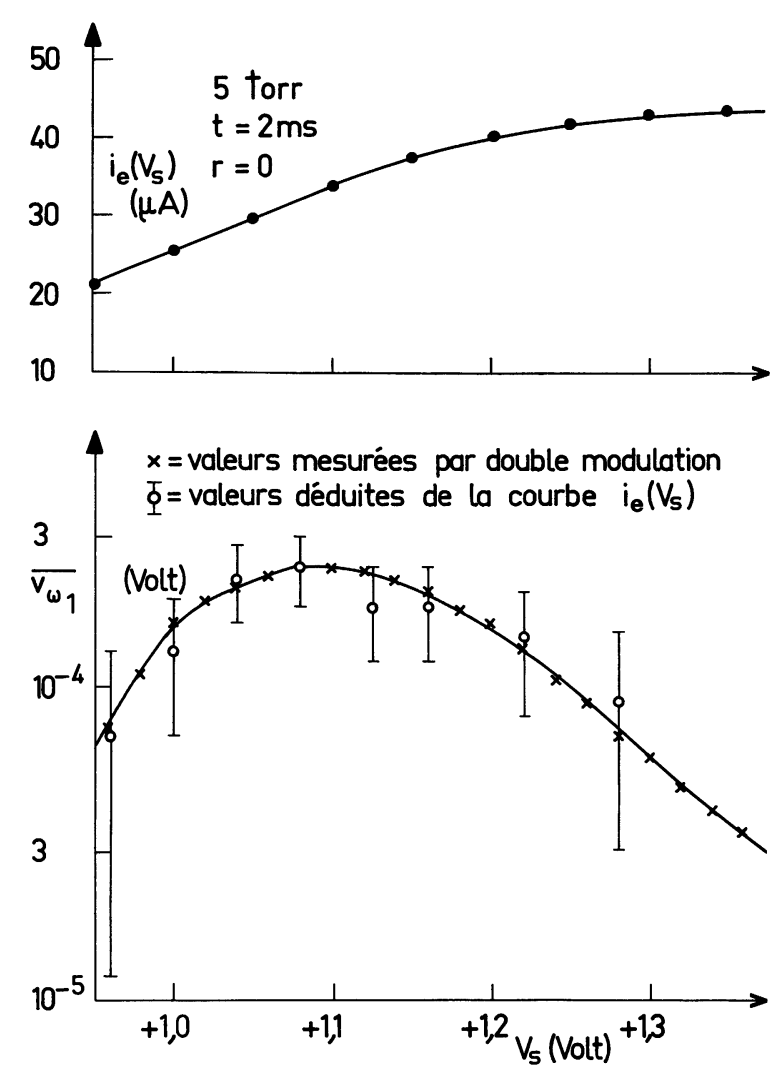

Fig. 6. - La partie supérieure de la figure montre la branche électronique de la caractéristique de sonde, mesurée sur l'axe de la cellule, à 5 torr et à $2 \mathrm{~ms}$ dans la post-luminescence. La partie inférieure montre la calibration du signal mesuré, par double différenciation graphique (cercles $\$$ ) et par la méthode de Druyvesteyn (croix $x$ ).

[The top part shows the electronic branch of the probe characteristic measured on the axis of the cell at 5 torr and $2 \mathrm{~ms}$ in the afterglow. The lower part shows the calibration of the measured signal, using the double graphic differentiation of the electronic branch (open circles $\Phi$ ) and the Druyvesteyn method (crosses $\times$ ).]

$$
\begin{aligned}
& \text { - par interférométrie et radiométrie U.H.F., } \\
& \text { - par spectrométrie de masse, } \\
& \text { - et par absorption et émission optique. }
\end{aligned}
$$

Des mesures par sonde à plus haute pression dans l'hélium et d'autres gaz sont sans doute possibles à condition de toujours respecter les limites de validité de la méthode $(\S 2)$. La première figure donne la fonction de distribution $f(\varepsilon)$ entre 4 et $20 \mathrm{eV}$, mesurée sur l'axe de la cellule $(r=0)$, à trois instants de la post-luminescence de l'hélium ionisé, avec une amplitude de modulation $V_{0}$ de $1 \mathrm{~V}$ et une résolution temporelle $\Delta t$ de $200 \mu$ s, imposées par la faiblesse des signaux à mesurer, de l'ordre du microvolt pour $\varepsilon=20 \mathrm{eV}$.

Ces courbes mettent en évidence un écart important à la fonction de Maxwell. En effet, dans ce domaine d'énergie la distribution mesurée est $10^{6}$ à $10^{10}$ fois plus peuplée que la maxwellienne.

On constate une décroissance rapide de cette partie haute énergie de la distribution en fonction du temps. Le nombre d'électrons, d'énergie comprise entre 4 et $20 \mathrm{eV}$, diminue en moyenne d'un facteur 5 entre 


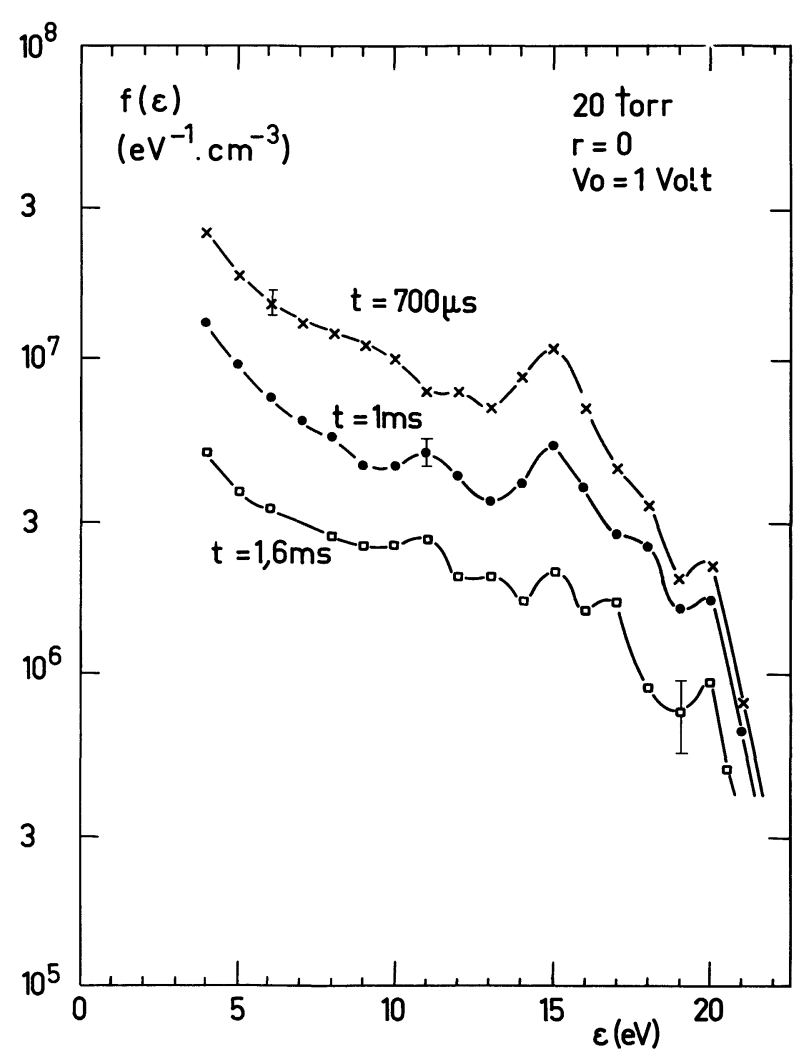

Fig. 7. - Fonctions de distribution en énergie, mesurées à 20 torr, suivant l'axe de la cellule, à différents instants de la post-décharge $(0,7 ; 1,0$ et $1,6 \mathrm{~ms})$.

[Electron energy distribution functions, measured at 20 torr, on the axis of the cell, at different times in the afterglow (0.7, 1.0 and $1.6 \mathrm{~ms})$.]

$700 \mu$ s et $1600 \mu \mathrm{s}$, alors que la densité électronique totale ne varie que d'un facteur 2 dans le même intervalle de temps.

Les barres d'erreur correspondent aux fluctuations du signal dues au bruit.

La figure 8 montre la variation spatiale de la fonction de distribution entre 4 et $20 \mathrm{eV}$, toujours à 20 torr, et à $1 \mathrm{~ms}$ dans la post-décharge. La forme des courbes est identique aux trois positions considérées. La décroissance de l'énergie quand on s'éloigne de l'axe de la cellule $(r=0)$ est clairement mise en évidence, elle rend presque impossible la détection des électrons d'énergie supérieure à $18 \mathrm{eV}$ pour $r=\frac{3}{4} R$.

A 5 torr, le libre parcours moyen des électrons $\lambda_{\mathrm{e}}$ est nettement supérieur au rayon de la sonde ce qui permet d'atteindre une portion de la maxwellienne suffisante pour en déduire la température électronique équivalente. Cette détermination est favorisée par la disparition très rapide par diffusion des électrons non thermiques au fur et à mesure de leur création. La figure 9 montre le signal de dérivée seconde $v_{\omega_{1}}$, mesuré à $500 \mu$ s dans la post-décharge, par la sonde centrale et la sonde située aux trois quarts du rayon, avec une très petite amplitude de modulation $\left(10^{-2} \mathrm{~V}\right)$ pour éviter toute distorsion. Les deux courbes présentent sur deux ordres de grandeur une décroissance

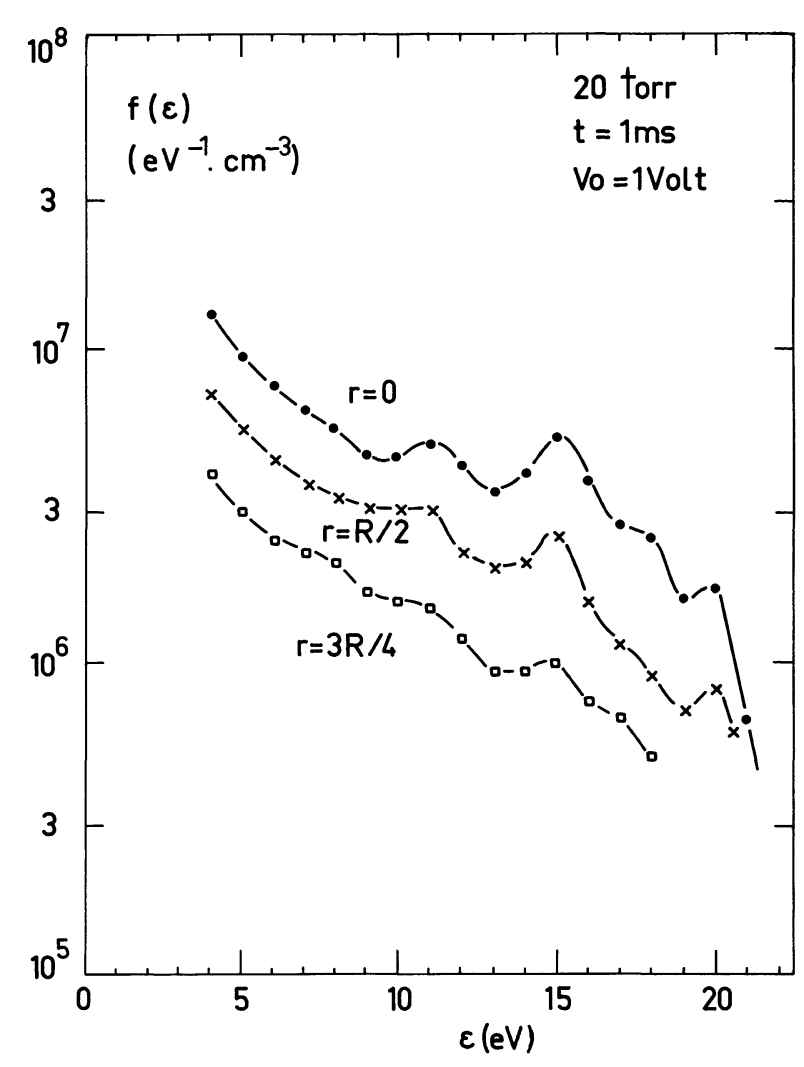

Fig. 8. - Fonctions de distribution en énergie, mesurées à 20 torr, à $1 \mathrm{~ms}$ dans la post-décharge, pour 3 positions de sonde.

[Electron energy distribution functions, measured at 20 torr and $1 \mathrm{~ms}$ in the afterglow, for three positions of the probe.]

exponentielle correspondant à une distribution de Maxwell

$\begin{array}{lll}\text { à } 395 \mathrm{~K} & \text { pour } & r=0 \\ \text { et } 300 \mathrm{~K} & \text { pour } & r=\frac{3}{4} R .\end{array}$

La température électronique équivalente de la partie maxwellienne de la fonction de distribution mesurée tend vers la température ambiante au voisinage des parois de l'enceinte.

Cette mesure ne correspond pas à la détermination complète de la partie maxwellienne de la distribution mais permet de suivre l'évolution de sa température équivalente.

5. Discussion et conclusion. - Nous avons montré que la distribution en énergie des électrons dans une post-décharge peut être déterminée par la méthode de la dérivée seconde, à l'aide de sondes de Langmuir, avec une bonne résolution temporelle, énergétique et spatiale, dans des conditions expérimentales de pression et de tension élevées.

Cette méthode s'applique en effet de façon très satisfaisante si l'on se limite, à forte pression, à la mesure de la partie haute énergie de la distribution.

C'est grâce à la très grande sélectivité du circuit de mesure, à la grande sensibilité du système de détection et à l'excellente linéarité de tous les composants 


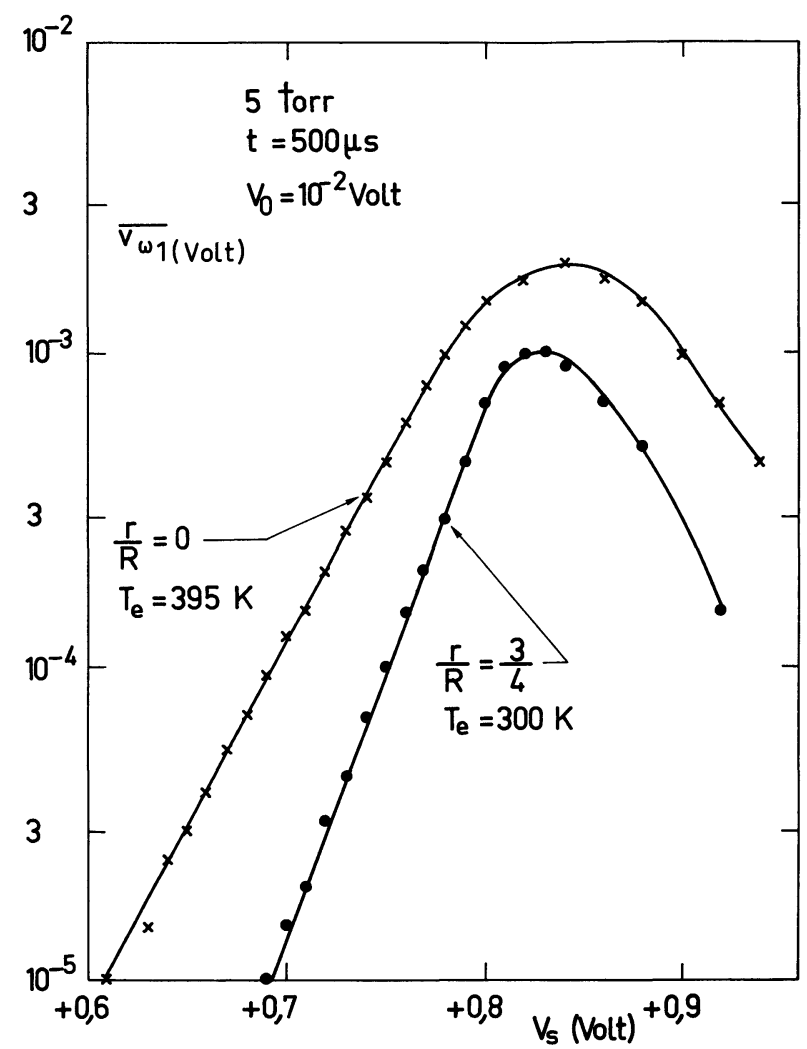

Fig. 9. - Détermination de la température électronique équivalente, à 5 torr et $500 \mu$ s dans la post-décharge.

[Determination of the equivalent electronic temperature at 5 torr and $500 \mu$ in the afterglow.]

électroniques que ces mesures ont pu être effectuées, en valeur absolue. Le minimum détectable de $10^{5}$ électrons par $\mathrm{cm}^{3}$ et par $\mathrm{eV}$ est de très loin la meilleure performance obtenue dans ce domaine.

Parmi les erreurs systématiques, minutieusement étudiées par Monchicourt [14], seule celle correspondant à la calibration de l'appareil de mesure est de quelque importance mais reste inférieure à $20 \%$. L'incertitude sur la surface de capture des sondes est évidemment prise en compte dans le calcul des barres d'erreur. Tous les effets secondaires tels que les collisions des métastables sur la sonde ou les effets photoélectriques n'ont aucune influence sur le signal mesuré puisqu'ils ne dépendent pas de la polarisation de la sonde. Seule la réflection des électrons sur la sonde peut légèrement modifier le signal mesuré dans un domaine de $0,5 \mathrm{eV}$ autour du potentiel plasma.

Les différentes perturbations créées par une sonde plongée dans un gaz ionisé ont été précédemment étudiées [13] de façon très approfondie. Dans nos conditions de mesure la formule de Druyvesteyn (1) s'applique avec une très bonne approximation, bien que le libre parcours moyen des électrons soit de l'ordre du diamètre de la sonde, le nombre d'électrons collectés reste en effet toujours petit devant le nombre d'électrons repoussés.

D'autres formules $[9,10]$ doivent être utilisées dans le cas contraire.

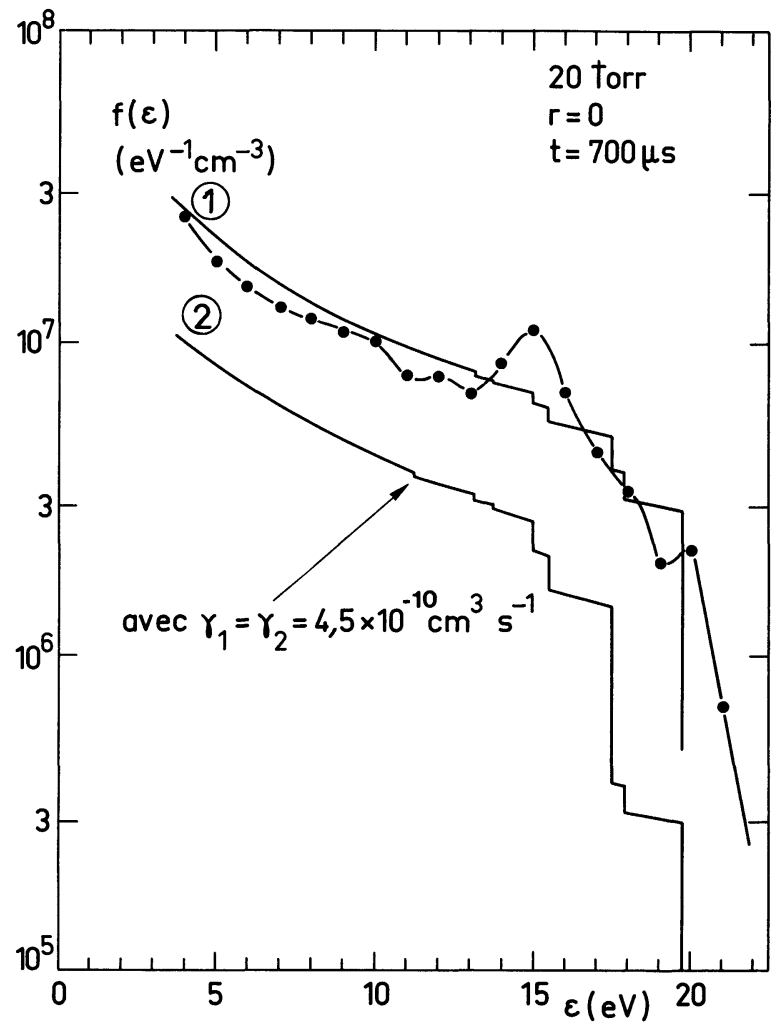

Fig. 10. - Comparaison théorie-expérience à 20 torr, suivant l'axe de la cellule expérimentale et à $700 \mu$ s dans la post-décharge. Les valeurs expérimentales de la fonction de distribution sont représentées par les points. La courbe (1) correspond à la fonction de distribution en énergie, calculée entre 4 et $20 \mathrm{eV}$, par le programme de simulation de l'évolution temporelle et spatiale de la postdécharge, avec l'ensemble des coefficients des taux de réaction publiés par Deloche et al. [3]. La courbe (2) est obtenue par le même calcul en ne modifiant que la valeur des coefficients des taux de désexcitation superélastique des métastables atomiques $\gamma_{1}$ et moléculaires $\gamma_{2}$.

[Comparison of computed curves (full lines) to experimental points (dots), at 20 torr, on the axis of the experimental chamber and at $700 \mu \mathrm{s}$ in the afterglow. Curve (1) gives the electron energy distribution function, between 4 and $20 \mathrm{eV}$, as calculated by the computer simulation of the helium afterglow, using the set of the rate coefficients published by Deloche et al. [3]. Curve (2) shows the same calculation with different values of the superelastic deexcitation rate coefficients of the atomic $\gamma_{1}$ and molecular $\gamma_{2}$ metastables.]

Etant donné l'importance du courant ionique il n'est pas possible de déterminer la dérivée seconde du courant électronique par double différenciation graphique ou électronique à partir des caractéristiques de sonde. Seule la méthode de Druyvesteyn permet cette mesure.

La comparaison des fonctions de distribution mesurées aux résultats de nos modèles théoriques sera publiée ultérieurement [7]. La figure 10 donne un exemple de la comparaison théorie-expérience et montre l'accord obtenu en prenant les valeurs des coefficients des taux de réaction publiés par Deloche et al. [3]. Cet excellent accord entre les valeurs calculées et expérimentales a permis la mesure directe des taux 
de production des électrons chauds et la confirmation de la validité de notre interprétation théorique $[1,2,3]$.

Il est important de souligner ici l'influence de la méthode de mesure sur la forme des distributions obtenues expérimentalement. Etant donné les valeurs absolues extrêmement faibles des courants d'électrons $i_{\omega_{1}}$ à mesurer, on ne peut prétendre obtenir les distributions avec une très grande résolution en énergie. L'amplitude de modulation $V_{0}$ dans toutes nos mesures ne peut facilement être fixée à moins de $1 \mathrm{~V}$. L'utilisation des formules (6) ou (8) est alors parfaitement justifiée si la fonction de distribution varie lentement, par exemple entre les énergies de création des électrons, faciles à observer à 15 ou $20 \mathrm{eV}$, car seule la dérivée seconde contribue au signal mesuré. $\mathrm{Si}$ au contraire la pente de $f(\varepsilon)$ varie très rapidement dans le domaine $\varepsilon-2 e V_{0}, \varepsilon+2 e V_{0}$, correspondant à l'amplitude maximale de la modulation $\Delta V$, alors toutes les dérivées paires de $i_{\omega_{1}}(V)$ (5) contribuent à la valeur du signal détecté et le développement limité de $i(V+\Delta V)$, en supposant $V$ petit, ne s'applique plus. Cette variation très rapide de $f(\varepsilon)$ se produit pour chaque énergie de création des électrons non thermiques.

Une interprétation rigoureuse des mesures de $i_{\omega_{1}}$ impose le calcul analytique du terme correspondant à la pulsation $\omega_{1}$, dans l'expression générale du courant collecté par la sonde, portée au potentiel $V+\Delta V:$

$$
\begin{aligned}
i(V+ & \Delta V)=\sqrt{\frac{2}{m}} \frac{e S}{4} \times \\
& \times \int_{e(V+\Delta V)}^{\infty}[\varepsilon-e(V+\Delta V)] \frac{f(\varepsilon)}{\varepsilon^{1 / 2}} \mathrm{~d} \varepsilon .
\end{aligned}
$$

Ces dérivées d'ordre supérieur à 2 ont tendance à créer une distorsion des courbes mesurées, lorsque l'amplitude de modulation n'est pas assez petite pour décrire correctement la variation de $f(\varepsilon)$. Il est important de bien distinguer ces distorsions, au niveau des énergies de création des électrons chauds, de la forme réelle de la distribution.

A notre connaissance, un seul autre dispositif expérimental $[5,6]$, basé sur le même principe, a précédemment été réalisé en régime pulsé. Il a été étudié pour déterminer la distribution en énergie des électrons à basse pression, de l'ordre du torr. Bien que la méthode de Druyvesteyn s'applique d'autant mieux que la pression est basse, l'interprétation des fonctions mesurées n'est pas forcément plus simple. En effet à basse pression, la diffusion des électrons créés à grande énergie devient très importante, la pente de $f(\varepsilon)$ entre deux énergies de création d'électrons non maxwelliens est très forte, la fonction de distribution ne peut être décrite, sans distorsion considérable, qu'avec une amplitude de modulation $V_{0}$ très petite, ce qui impose une sensibilité très grande. L'interprétation des courbes obtenues par Blagoev et al. $[5,6,15]$ est donc plus difficile que dans nos conditions expérimentales, d'autant plus que la sensibilité de leur système de mesure est environ cinquante fois inférieure à celle de notre dispositif, avec une amplitude de modulation de $1 \mathrm{~V}$. D'autre part le schéma de leur circuit électronique $[5,6]$ indique un commutateur placé en série dans le circuit de sonde, c'est-à-dire une modulation $\Delta V . f(t)$ appliquée uniquement pendant le temps $\Delta t$ de l'ouverture de la porte de mesure, ce qui est apparemment incompatible avec la méthode d'échantillonnage présentée, qui correspond à une modulation $\Delta V$ appliquée en permanence sur la sonde.

Le dispositif expérimental que nous venons de présenter permet grâce à ses caractéristiques et à ses performances, de mesurer en valeur absolue, pour la première fois à des pressions de quelques dizaines de torr, avec une précision de l'ordre de $20 \%$, les variations temporelles et spatiales de la fonction de distribution en énergie des électrons au cours d'une postdécharge répétitive.

Ce dispositif, utilisé dans l'hélium ionisé, permet également la détermination expérimentale de l'énergie des électrons dans toute décharge pulsée de n'importe quel gaz ionisé et dans un très large domaine de conditions expérimentales.

La mesure de la fonction de distribution en énergie des électrons est un diagnostic fondamental en physique des gaz ionisés, il est important dans les décharges continues et indispensable à la compréhension des mécanismes élémentaires qui gouvernent l'évolution des post-luminescences. Il conduit, entre autres, à déterminer l'écart à l'équilibre thermodynamique des électrons, à mesurer l'élévation de la température équivalente de la partie maxwellienne des distributions, à préciser quels sont les mécanismes de chauffage et de refroidissement prépondérants, à définir le rôle de la diffusion, et enfin à mesurer directement les coefficients des taux de création des électrons non maxwelliens.
[1] Wells, W. E., Monchicourt, P., Deloche, R. et BerLANDE, J., Phys. Rev. A 8 (1973) 381.

[2] Monchicourt, P., Touzeau, M. et Wells, W. E., J. Physique Colloq. 34 (1973) C2-145.
[3] Deloche, R., Monchicourt, P., Cheret, M. et Lambert, F., Phys. Rev. A 13 (1976) 1140.

[4] Deloche, R., Wells, W. E., Monchicourt, P. et BerLANDE, J., J. Phys. B. B 6 (1973) 1881. 
[5] Blagoev, A. B., Kolokolov, N. B. et Milenin, V. M., Sov. Phys.-Techn. Phys. 17 (1973) 1359.

[6] Blagoev, A. B., Kagan, Y. M., Kolokolov, N. B. et LyaGUSHCHENko, R. I., Sov. Phys.-Techn. Phys. 19 (1974) 211.

[7] Monchicourt, P., Deloche, R., soumis au J. Phys. B.

[8] Druyvesteyn, M. J., Z. Phys. 64 (1930) 781.

[9] Wasserstrom, E., Su, C. H. and Probstein, P. F., Phys. Fluids 8 (1965) 56.

Chou, Y. S., Talbot, L. and Willis, D. R., Phys. Fluids 9 (1966) 2150.

Bienkowski, G. H. and Chang, K. W., Phys. Fluids 11 (1968) 784.

LeEs, L., Memorandum no 51, California Inst. of Techn. (1959).

Su, C. H. and LaM, S. H., Phys. Fluids 6 (1963) 1479.

CoHen, I. M., Phys. Fluids 6 (1963) 1492.
Davydov, B. and Zmanovakaja, L., Zh. Teckh. Fiz. 7 (1936) 1244.

Waymouth, J. F., Phys. Fluids 7 (1964) 1843.

[10] Case, C. T., Tenth Conf. on Pheno. in Ion. Gases (Oxford) 1970, p. 402.

[11] Sloane, H. R. et McGregor, E. I. R., Phil. Mag. 18 (1934) 193.

Boyd, R. L. F. et Twiddy, N. D., Proc. Roy. Soc. 250 (1959) 53.

Kaggan, Y. M., Kolokolov, N. B. et Milenin, V. M., Sov. Phys.-Techn. Phys. 13 (1969) 1468.

[12] Ahrweiller, M., Nucl. Instrum. Methods 96 (1971) 205.

[13] SwIFT, J. D. et SchwAR, M. J. R., Electrical probes and plasma diagnostics, London ILIFFE Books L.T.D. (1970).

[14] Monchicourt, P., Thèse de Doctorat d'Etat. Université Paris-Sud no 1877, 30 septembre 1977.

[15] Blagoev, A. B., Kagan, Y. M., Kolokolov, N. B. et LyaGUSHCHenko, R. I., Sov. Phys.-Techn. Phys. 29 (1975) 360. 